\title{
Asteroseismology of the planet-hosting star $\mu$ Arae
}

\section{The acoustic spectrum ${ }^{\star}$}

\author{
F. Bouchy ${ }^{1,2}$, M. Bazot ${ }^{3}$, N. C. Santos ${ }^{4,5}$, S. Vauclair ${ }^{3}$, and D. Sosnowska ${ }^{5}$ \\ 1 Laboratoire d'Astrophysique de Marseille, Traverse du Siphon, 13013 Marseille, France \\ e-mail: Francois.Bouchy@oamp.fr \\ 2 Observatoire de Haute Provence, 04870 St. Michel l'Observatoire, France \\ 3 Université Paul Sabatier, Observatoire Midi-Pyrénées, 14 Av. E. Belin, 31400 Toulouse, France \\ ${ }^{4}$ Lisbon Observatory, Tapada da Ajuda, 1349-018 Lisboa, Portugal \\ 5 Observatoire de Genève, 51 Ch. des Maillettes, 1290 Sauverny, Switzerland
}

Received 13 January 2005 / Accepted 4 March 2005

\begin{abstract}
We present HARPS spectroscopy of $\mu$ Arae (HD 160691) performed for studying the origin of the metallicity excess in this planet-hosting stars. The asteroseismologic campaign led to the previously reported discovery of a 14 earth mass planetary companion (Santos et al. 2004b, A\&A, 426, L19). The present analysis reinforces this interpretation by excluding other possible processes for explaining the observed Doppler variation and leads to the identification of up to $43 p$-mode oscillations with $l=0-3$, frequencies in the range $1.3-2.5 \mathrm{mHz}$ and amplitudes in the range $10-40 \mathrm{~cm} \mathrm{~s}^{-1}$.
\end{abstract}

Key words. stars: individual: $\mu$ Arae - stars: oscillations - techniques: radial velocities - stars: planetary systems

\section{Introduction}

One particularly important fact about planet-hosting stars is helping astronomers to understand the mechanisms of planet formation and evolution. These stars were found to be particularly metal-rich, i.e. they have, on average, a metal content higher by $\sim 0.2$ dex than in field stars without detected planetary companions (e.g. Gonzalez 1998; Santos et al. 2001, 2003, 2004a).

If stars with planets were formed out of more-than-average metallicity material, then metallicity might play a key role in the formation of giant planets. However some evidence suggests that planetary material might have fallen onto the planethosting stars (Israelian et al. 2001; Murray \& Chaboyer 2002) and might be able to produce the metallicity excess observed. In this case result in the planet migration may being engulfed by the star. Which of these two scenarios is correct? Is the metallicity excess observed in planet-hosting stars of primordial origin, or is it due to stellar pollution effects?

Asteroseismology consists in measuring properties of $p$-mode oscillations and provides a unique tool for "drilling" stellar interiors and for testing the internal structure and chemical composition of bright solar-type stars (e.g. Bouchy \& Carrier 2003; Bedding \& Kjeldsen 2003). It also appears as

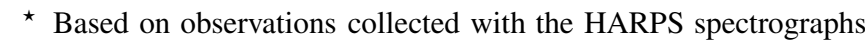
at the 3.6-m telescope (La Silla Observatory, ESO, Chile: program 073.D-0578). the only means to determine the metallicity gradient across the stellar interior in order to solve the question of the origin of metallicity excess in planet-hosting stars (Vauclair 2004; Bazot \& Vauclair 2004).

We noted earlier (Bouchy et al. 2004; Bouchy 2005) that with a radial velocity precision bellow $1 \mathrm{~m} \mathrm{~s}^{-1}$, the HARPS spectrograph (Pepe et al. 2002; Mayor et al. 2003) mounted at the 3.6-m telescope offered promising perspectives for such asteroseismologic studies. We present here results of our asteroseismologic campaign obtained with this instrument on HD 160691, one of the most metal-rich stars with planetary companions. Comparison with stellar structure models and interpretation are presented in a companion paper (Bazot et al. 2005).

\section{Observations and data reduction}

HD 160691 ( $\mu$ Ara, HR 6585) is a G3IV-V star with a $V$ magnitude of 5.1 and a metallicity $[\mathrm{Fe} / \mathrm{H}]$ of +0.32 . A giant planet $\left(1.7 M_{\text {Jup }}\right)$ in a long period (637 days) orbit was detected by Butler et al. (2001). The orbital solution was updated by Jones et al. (2002) and Gozdziewski et al. (2003), who found a long-term drift, possibly due to the presence of a second body ( $>1.5 M_{\text {Jup }}, \sim 1500$ days) in the system. Bright enough for HARPS, this target is furthermore well suited for an asteroseismologic campaign (observable more than $10 \mathrm{~h}$ per night). HD 160691 was observed over 8 nights in June 2004. 
Table 1. Journal of radial velocity measurements. The minimum and maximum $S / N$ of spectra obtained during the night is given. The dispersion $\sigma_{\text {rms }}$ is strongly dominated by the seismic signal and do not reflect the precision of individual points.

\begin{tabular}{ccccc}
\hline \hline Date & Nb spectra & Nb hours & $S / N$ & $\sigma_{\text {rms }}\left[\mathrm{m} \mathrm{s}^{-1}\right]$ \\
\hline $2004 / 06 / 03$ & 279 & 10.32 & $105-181$ & 2.49 \\
$2004 / 06 / 04$ & 274 & 10.19 & $65-237$ & 1.71 \\
$2004 / 06 / 05$ & 285 & 10.34 & $77-239$ & 1.79 \\
$2004 / 06 / 06$ & 286 & 10.38 & $132-235$ & 2.03 \\
$2004 / 06 / 07$ & 275 & 10.35 & $43-263$ & 2.20 \\
$2004 / 06 / 08$ & 229 & 8.43 & $24-233$ & 2.04 \\
$2004 / 06 / 09$ & 202 & 7.43 & $57-147$ & 1.65 \\
$2004 / 06 / 10$ & 274 & 10.42 & $32-186$ & 1.49 \\
\hline
\end{tabular}

We took sequences of 100-s exposures with a dead time of 31-s in-between. In total, 2104 spectra were collected with typical night-averaged signal-to-noise ratios $(S / N)$ per pixel in the range $95-193$ at $550 \mathrm{~nm}$.

The spectra obtained were extracted on-line and in realtime using the HARPS pipeline. Wavelength calibration was performed with ThAr spectra. The radial velocities were obtained by weighted cross-correlation with a numerical mask constructed from the Sun spectrum atlas. We also computed off-line the stellar radial velocities using the optimum weight procedure as described by Bouchy et al. (2001) but found no significant gain. The journal of the observations is given in Table 1 and the radial velocities are presented in Figs. 1 and 2. The dispersion of each individual night, in the range $1.5-2.5 \mathrm{~m} \mathrm{~s}^{-1}$, is strongly dominated by the acoustic modes with a period around 8 min, as shown in Fig. 3 .

The simultaneous ThAr spectrum was used to compute the instrumental drift using the optimum weight procedure. The spectrograph drift was systematically lower than $0.75 \mathrm{~m} \mathrm{~s}^{-1}$ during the night and lower than $2 \mathrm{~m} \mathrm{~s}^{-1}$ during the whole campaign, illustrating the extreme stability of HARPS. We observed without simultaneous Thorium on the fourth night to construct, without any risk of ThAr contamination, a high $S / N$ combined spectrum for spectroscopic analysis of HD 160691.

\section{A low-mass-planetary companion}

Our first surprise was to detect an unexpected modulation in the Doppler signal with a semi-amplitude of about $4 \mathrm{~m} \mathrm{~s}^{-1}$ and a period equal to or greater than 8 days. Figure 1 clearly shows that we would not have been able to detect such a small signal if only one measurement was made each night. Indeed, the semiamplitude of $4 \mathrm{~m} \mathrm{~s}^{-1}$ corresponds typically to the peak-to-peak variation induced by the $p$-modes in the time series.

In order to confirm the long period modulation, further observations were carried on during two GTO runs (program 073.C-0005) in July and August 2004. Considering that the longest periods of $p$-modes in this star are around $11 \mathrm{~min}$ (see Sect. 4), we decided to construct each measurement by averaging consecutive independent observations over a period

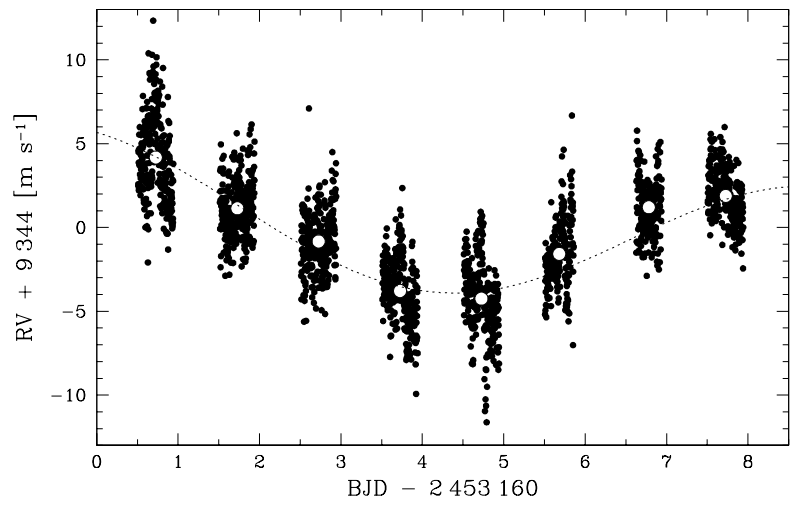

Fig. 1. Radial velocity measurements of HD 160691 . White circles correspond to the night average. The dotted curve correspond to the orbital fit of the low-mass-planetary companion confirmed through additional measurements by Santos et al. (2004).

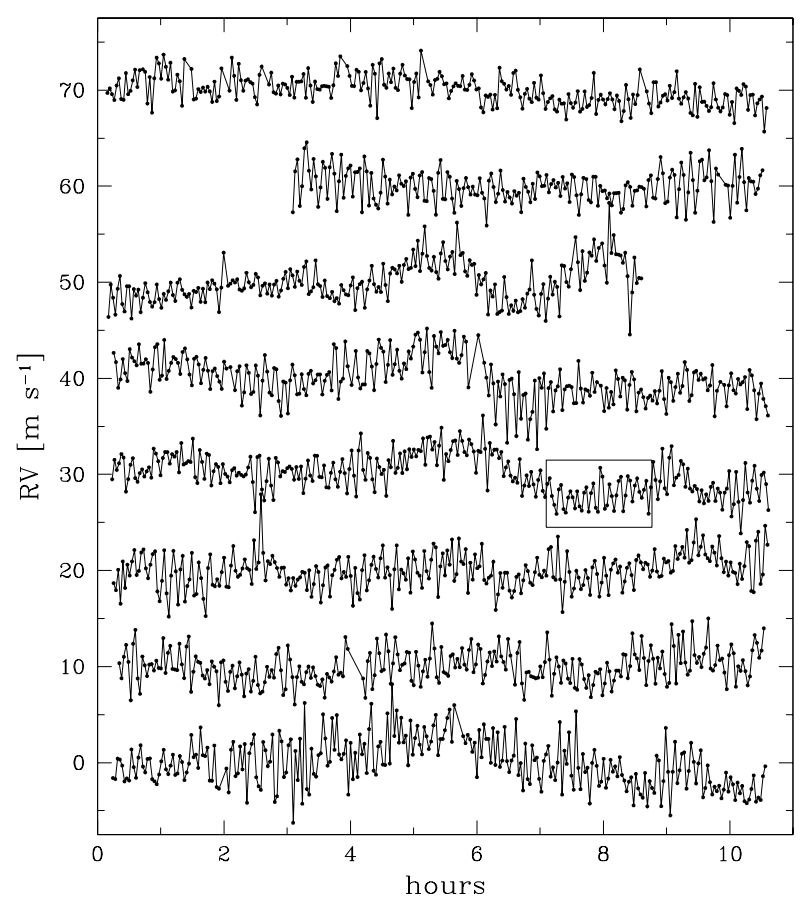

Fig. 2. Radial velocity time series of individual nights displayed from bottom to top to a relative scale. The box on the fourth night is expanded in Fig. 3.

of twice the longest acoustic period ( $22 \mathrm{~min})$. These further measurements allowed us to confirm the modulation and determine the period to 9.55 days. This Doppler modulation was interpreted as caused by a 14 earth mass planetary companion, the third one detected in this exoplanetary system and the lightest planetary companion ever observed (Santos et al. 2004b).

In order to verify if this long period modulation was due to a blended binary system or a stellar spot, we conducted a bisector analysis of the Cross-Correlation Function (CCF) as described in Queloz et al. (2001) and Santos et al. (2002). Such an analysis is very efficient in discriminating between radialvelocity variations due to changes in the spectral-line profiles from pure Doppler variations due to the orbital motion of the star. Figure 4 presents a typical CCF of HD 160691. We computed the bisector velocity of each CCF for 100 different levels 


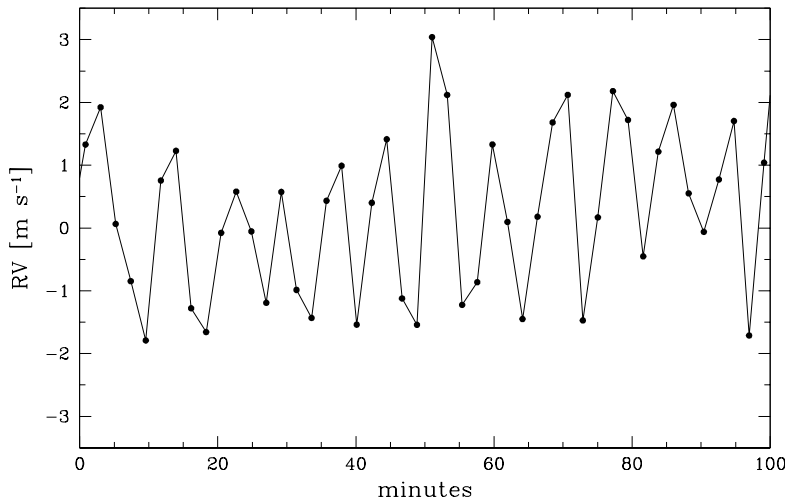

Fig. 3. Zoom of radial velocity measurements showing the presence of $p$-modes in the time series with periods of around $8 \mathrm{~min}$. The semiamplitude of about $2 \mathrm{~m} \mathrm{~s}^{-1}$ does not represent the individual amplitude of $p$-modes but comes from the interference of several modes.

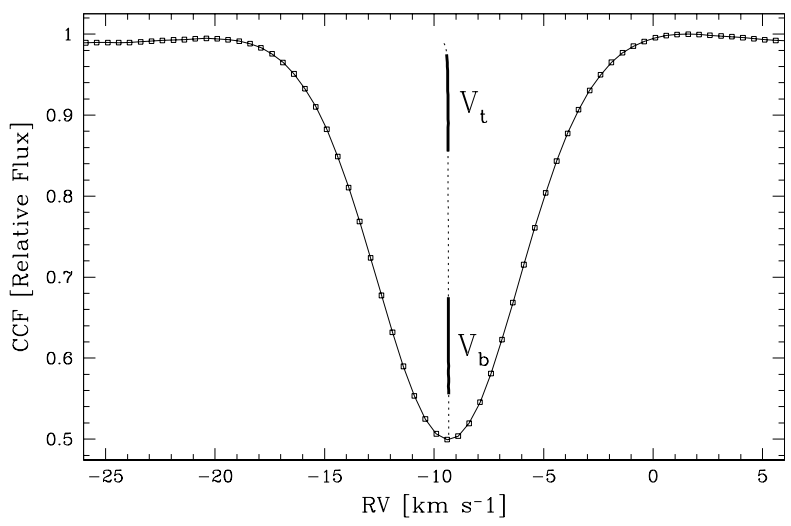

Fig. 4. Typical Cross Correlation Function of HD 160691 with location of the intervals used to compute the bisector velocity span $V_{\mathrm{t}}-V_{\mathrm{b}}$.

(dividing the CCF in 100 slices). We defined the "bottom" velocity $V_{\mathrm{b}}$ and "top" velocity $V_{\mathrm{t}}$ by averaging the values in the range $10-35 \%$ and $70-95 \%$ of the CCF depth, respectively. The difference $V_{\mathrm{t}}-V_{\mathrm{b}}$ is equivalent to the bisector velocity span and is used to measure the variations of the line profile. The result of our bisector analysis is presented in Fig. 5 at the same scale as Fig. 1. The dispersion of the velocity span on an individual night is about $2 \mathrm{~m} \mathrm{~s}^{-1}$. No variation appears in phase with the radial-velocity variations of period of 9.55 days. We checked that no high frequency signals are present in the velocity span showing that $p$-modes induce a pure Doppler variation on the spectral lines. The dispersion of the night-averaged-velocity span is $0.8 \mathrm{~m} \mathrm{~s}^{-1}$. This result indicates that the periodic-radialvelocity change is not due to an blended background binary (as HD 41004 - see Santos et al. 2002) nor a dark photospheric stellar spot (as HD 166435 - see Queloz et al. 2001).

We also checked the magnetic activity of HD 160691 by computing the chromospheric activity index $S$ based on the relative flux level on CaII $\mathrm{H}$ and $\mathrm{K}$ lines (see Fig. 6). We measured the flux in two $1 \AA$ pass bands centered on the $\mathrm{H}$ and $\mathrm{K}$ lines normalized by two $20 \AA$ wide sections of photospheric flux centered at 3900 and $4000 \AA$. The index $S$, computed on the 2104 available spectra, has values in the range $0.120-0.125$. Once in the Mount Wilson scale (Vaughan et al. 1978), this

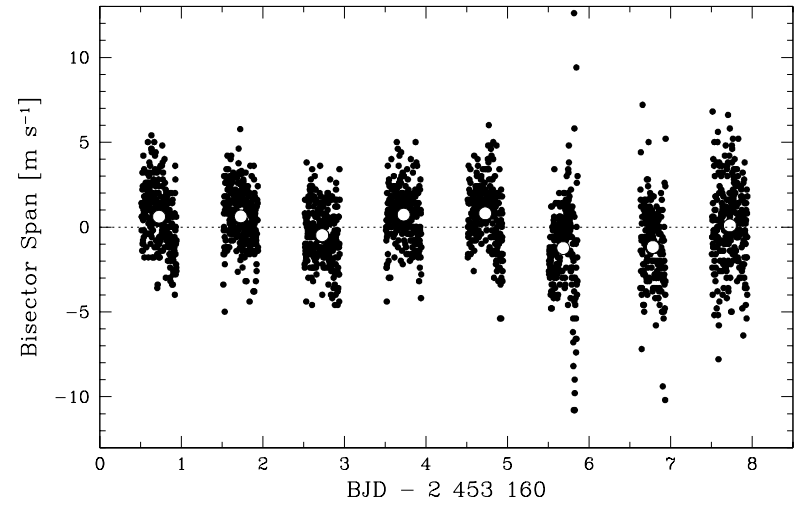

Fig. 5. Bisector velocity span of the CCF profile of HD 160691. White circles correspond to the night average.
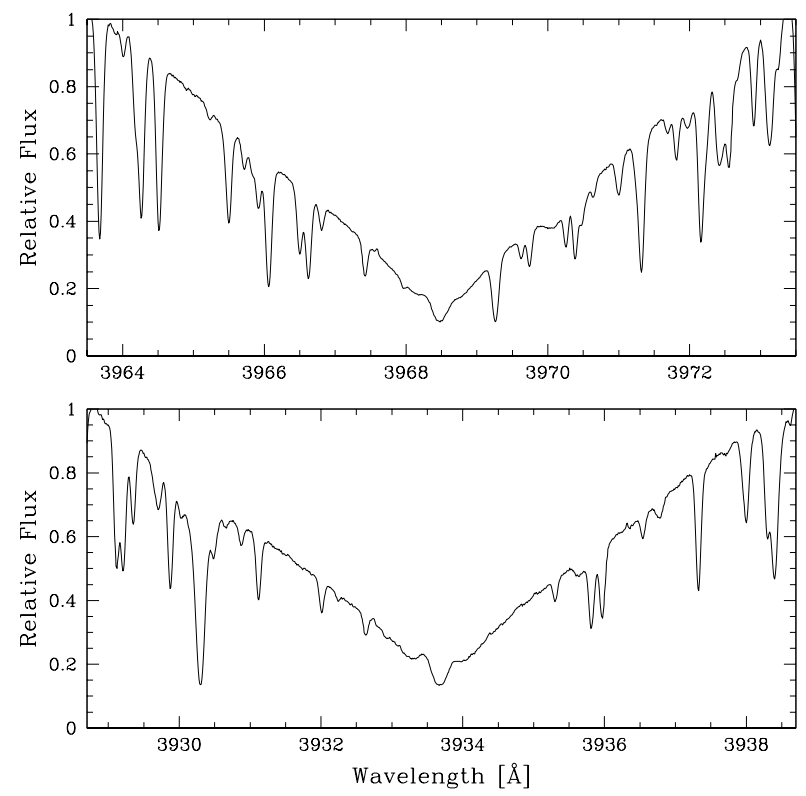

Fig. 6. CaII H and K lines for HD 160691 showing no chromospheric activity.

corresponds to a $\log R_{H K}^{\prime}=-5.034 \pm 0.006$ (Noyes et al. 1984). As mentioned by Santos et al. (2004b) this value, in close agreement with the one obtained by Henry et al. (1996), is typical of an inactive solar type star.

Finally our acoustic spectrum analysis (see Sect. 4) allows us to estimate the rotational period at $\sim 22$ days which definitively eliminates any stellar activity origin in the 9.55 day signal.

We noticed that the residuals around the best Keplerian fit have a rms of $0.43 \mathrm{~m} \mathrm{~s}^{-1}$ for the 8 night-averaged measurements made during our asteroseismologic campaign (average of more than 200 individual observations) and have a rms of $1.3 \mathrm{~m} \mathrm{~s}^{-1}$ for the other nights (average of 15 observations). This is due to the presence of low frequency (few hours) modulations in the Doppler signal (see Fig. 2) with semi-amplitudes of $1-2 \mathrm{~m} \mathrm{~s}^{-1}$ which are not averaged by 15 consecutive independent observations. We checked that this noise did not appear in the Thorium signal. We also checked that this noise was not introduced by a chromatic effect on the fiber entrance due to atmospheric-dispersion-correction residuals with change of 
seeing or change of fiber centering. The flux ratio between blue and red spectral orders do not present a correlation with the low frequency modulation seen in the Doppler signal. The origin of this noise is still unclear, but considering that it does not seem to be an instrumental effect and that its amplitude change with time, we strongly suspect that it could have a stellar origin (like granulation noise).

A third exoplanet with a low mass $\left(14 M_{\oplus}\right)$ and short period (9.55 days) was not completely unexpected and was considered as a possible chance by-product of this asteroseismologic campaign. Statistics of the exoplanet sample show that the probability of finding a new exoplanet is higher around a planet-hosting star (more than $10 \%$ of known exoplanets are in a multiple system), even if the host star is overmetallic. We show here that the only way to find such a companion with a semi-amplitude of only $4.1 \mathrm{~m} \mathrm{~s}^{-1}$ is to perform such an intensive campaign of radial velocity measurements in order to average properly and completely the stellar oscillations.

\section{Acoustic spectrum analysis}

In order to compute the power spectrum of the velocity time series of Fig. 1 (corrected for the orbital motion), we used the Lomb-Scargle modified algorithm (Lomb 1976; Scargle 1982) for unevenly spaced data. The resulting LS periodogram, shown in Fig. 7, exhibits a series of peaks between 1.3 and $2.5 \mathrm{mHz}$ modulated by a broad envelope, which is the typical signature of solar-like oscillations. This signature also appears in the power spectrum of each individual night. The mean white noise level computed in the range $0.7-1.2 \mathrm{mHz}$ is $2.6 \times$ $10^{-3} \mathrm{~m}^{2} \mathrm{~s}^{-2}$. Considering Gaussian noise, the mean noise level in the amplitude spectrum is $4.5 \mathrm{~cm} \mathrm{~s}^{-1}$. Considering that the time series is based on 2104 measurements, the corresponding velocity accuracy thus corresponds to $1.17 \mathrm{~m} \mathrm{~s}^{-1}$. The uncertainty due to photon noise is estimated to be $0.52 \mathrm{~m} \mathrm{~s}^{-1}$ indicating that we are not photon-noise limited. The origin of the external noise level of $\sim 1 \mathrm{~m} \mathrm{~s}^{-1}$ is still unclear. The main instrumental limitation of HARPS could come from the guiding and centering errors on the optical fiber input but tests made during commissioning indicated error sources at the level of $0.2 \mathrm{~m} \mathrm{~s}^{-1}$ (Mayor et al. 2003). We strongly suspect a stellar origin for this noise (like granulation noise).

In solar-like stars, $p$-mode oscillations are expected to produce a characteristic comb-like structure in the power spectrum with mode frequencies $v_{n, l, m}$ reasonably well approximated by the simplified asymptotic relation (Tassoul 1980):

$v_{n, l, m} \approx \Delta v \cdot\left(n+\frac{l}{2}+\epsilon\right)-\delta v_{02} \cdot \frac{l(l+1)}{6}-\Omega \cdot m$.

The two quantum numbers $n$ and $l$ correspond to the radial order and the angular degree of the modes, respectively. For stars for which the disk is not resolved, only the lowest-degree modes $(l \leq 3)$ can be detected. In case of stellar rotation, $p$-modes need to be characterized by a third quantum number $m$ called the azimuthal order $(-l \leq m \leq l)$. Quantities $\Delta v$, $\delta v_{02}$ and $\Omega$ correspond to the large spacing, the small spacing and the rotational splitting, respectively.

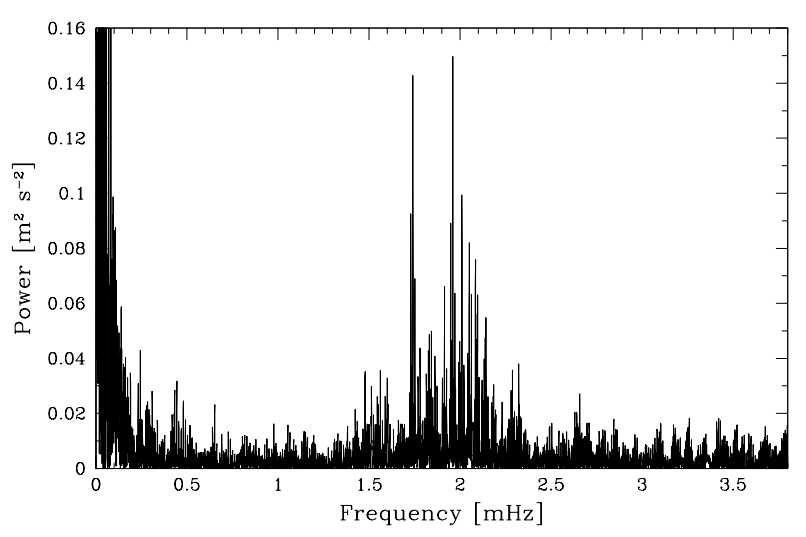

Fig. 7. Power spectrum of radial velocity measurements of HD 160691.

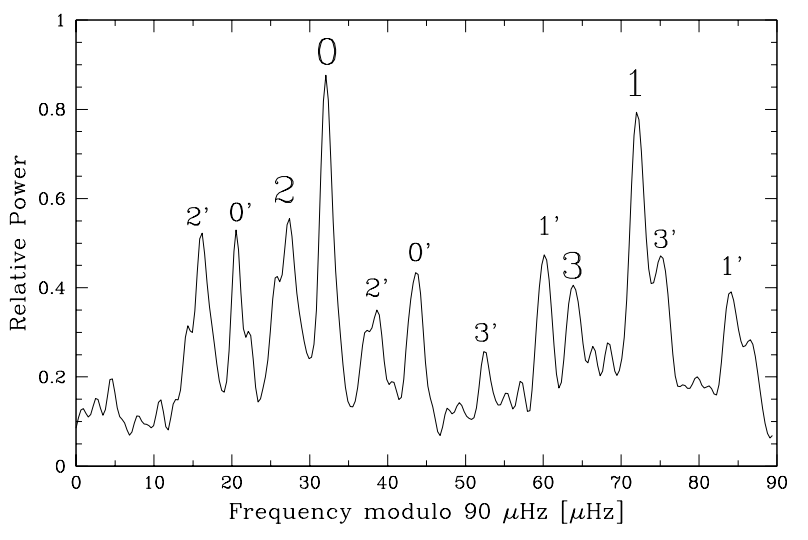

Fig. 8. Sum of the echelle diagram showing the modes $l=0,1,2,3$ and their side-lobes.

The power spectrum, shown in Fig. 7, presents a clear and unambiguous periodicity of $90 \mu \mathrm{Hz}$ both on its autocorrelation or in the comb response. In order to identify the angular degree $l$ of each mode individually, we divided the power spectrum into slices of $90 \mu \mathrm{Hz}$ and summed them. The result, which corresponds to the sum of the echelle diagram, is presented in Fig. 8 and allows us to unambiguously identify modes $l=0,1,2,3$ and their side-lobes due to the daily aliases at $\pm 11.57 \mu \mathrm{Hz}$.

We selected the strongest peaks with a signal-to-noise ratio greater than 3 in the amplitude spectrum and identified them as $p$-modes with $n$ value based on the asymptotic relation (see Eq. (1)) assuming that the parameter $\epsilon$ is near the solar value $\left(\epsilon_{\odot} \sim 1.5\right)$. The frequency and amplitude of these $33 p$-modes are listed in Tables 2 and 3. The amplitude was determined by assuming that none of the $p$-modes are resolved and corresponds to the height of the peak in the power spectrum after quadratic subtraction of the mean noise level. Considering that the frequency resolution of our time series is $1.56 \mu \mathrm{Hz}$, we adopted an uncertainty on no-resolved oscillation modes of $0.78 \mu \mathrm{Hz}$. Such an uncertainty can be considered as conservative in the case of infinite time-life modes with high signal-to-noise ratio $(\geq 3.5)$. A second inspection of the power spectrum with selection of peaks with signal-to-noise ratio in the range 2.5-3 (with amplitude in the range 11-13.5 $\mathrm{cm} \mathrm{s}^{-1}$ ) allowed us to propose 10 additional $p$-modes. We are aware 
Table 2. $P$-mode frequencies for HD $160691(\mu \mathrm{Hz})$. The frequency resolution of the time series is $1.56 \mu \mathrm{Hz}$. Frequencies with label ${ }^{a}$ correspond to peaks with a signal-to-noise ratio in the range $2.5-3$ and have to be considered with caution.

\begin{tabular}{ccccc}
\hline \hline & $l=0$ & $l=1$ & $l=2$ & $l=3$ \\
\hline$n=13$ & $\ldots$ & $\ldots$. & $1381.8^{a}$ & $\ldots$. \\
$n=14$ & $\ldots$. & 1424.5 & $\ldots$. & $\ldots$. \\
$n=15$ & 1478.4 & 1513.7 & $1555.1^{a} / 1557.0^{a}$ & $1592.9^{a}$ \\
$n=16$ & 1562.3 & 1600.2 & $\ldots$. & $1682.5^{a}$ \\
$n=17$ & $\ldots$. & $\ldots$. & $1731.8 / 1734.9$ & 1772.2 \\
$n=18$ & 1740.9 & 1780.8 & $1824.2 / 1826.6$ & 1862.3 \\
$n=19$ & 1831.6 & $\ldots$. & 1914.5 & 1954.7 \\
$n=20$ & 1922.0 & 1960.4 & $2005.9 / 2007.8$ & 2042.5 \\
$n=21$ & 2010.1 & 2051.0 & $2094.9 / 2096.7$ & 2132.8 \\
$n=22$ & 2101.0 & 2140.8 & $2182.9 / 2184.9$ & $\ldots$. \\
$n=23$ & $2189.7^{a}$ & 2231.0 & $2274.4^{a} / 2276.0^{a}$ & $2315.7^{a}$ \\
$n=24$ & 2280.3 & 2322.7 & $\ldots$. & $\ldots$. \\
$n=25$ & $\ldots$. & $\ldots$. & $\ldots$. & $\ldots$. \\
$n=26$ & $\ldots$. & $2504.5^{a}$ & $\ldots$. & $\ldots$. \\
\hline
\end{tabular}

Table 3. $P$-mode amplitudes for HD $160691\left(\mathrm{~cm} \mathrm{~s}^{-1}\right)$.

\begin{tabular}{|c|c|c|c|c|}
\hline & $l=0$ & $l=1$ & $l=2$ & $l=3$ \\
\hline$n=13$ & $\ldots$ & $\ldots$. & 11 & $\ldots$. \\
\hline$n=14$ & $\ldots$. & 14 & $\ldots$ & $\ldots$ \\
\hline$n=15$ & 18 & 16 & $10 / 11$ & 12 \\
\hline$n=16$ & 18 & 17 & $\ldots$. & 12 \\
\hline$n=17$ & $\ldots$. & $\ldots$ & $18 / 15$ & 13 \\
\hline$n=18$ & 38 & 21 & $16 / 21$ & 19 \\
\hline$n=19$ & 22 & $\ldots$ & 26 & 22 \\
\hline$n=20$ & 14 & 39 & $18 / 16$ & 19 \\
\hline$n=21$ & 32 & 29 & $24 / 25$ & 19 \\
\hline$n=22$ & 16 & 16 & $15 / 16$ & $\ldots$ \\
\hline$n=23$ & 12 & 15 & $12 / 11$ & 11 \\
\hline$n=24$ & 16 & 18 & $\ldots$ & $\ldots$ \\
\hline$n=25$ & $\ldots$ & $\ldots$ & $\ldots$ & $\ldots$ \\
\hline$n=26$ & $\ldots$ & 12 & $\ldots$. & $\ldots$ \\
\hline
\end{tabular}

that some of these weaker modes could be mis-identifications and should be considered with caution and lower confidence.

All the identified modes are displayed on the power spectrum in Fig. 9 and in the echelle diagram in Fig. 10. A few peaks with signal-to-noise greater than 3 but not identified as $p$-modes or side-lobes are displayed in Fig. 10 as crosses. This could indicate that some modes are split due to their damping time.

The large spacings $\Delta v=v_{n+1, l}-v_{n, l}$ were computed for each pair of consecutive modes with the same angular degree. For the $l=2$ modes, which are split into 2 components due to the stellar rotation, we used the average value of the 2 frequencies. The result is displayed in Fig. 11. Neglecting the first three points, the residual of the large separations after a third order polynomial fit is $1.04 \mu \mathrm{Hz} \mathrm{rms}$. This value indicates that the adopted uncertainty of $0.78 \times \sqrt{2}=1.1 \mu \mathrm{Hz}$ for the large separation is appropriate. The small spacings $\delta v_{0,2}=v_{n+1,0}-v_{n, 2}$ and $\delta v_{1,3}=v_{n+1,1}-v_{n, 3}$ are displayed in Fig. 12. The average
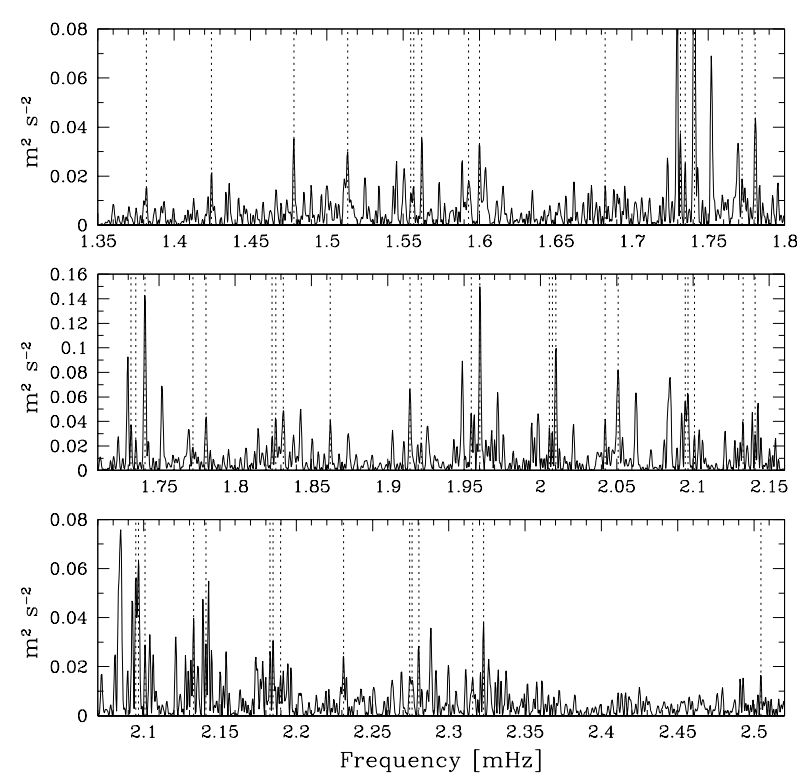

Fig. 9. Power spectrum of HD 160691 with the identified frequencies in Table 2 marked by dotted lines.

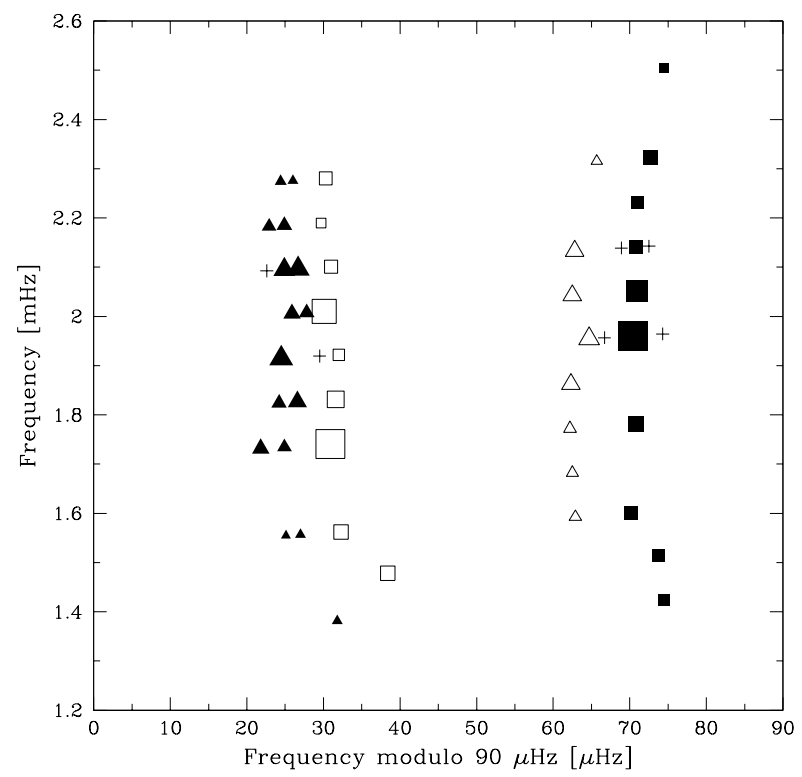

Fig. 10. Identified $p$-modes $l=0(\square), l=1(\mathbf{\square}), l=2(\mathbf{\Delta})$, and $l=3(\triangle)$ in echelle diagram format. The size of the symbols are proportional to the amplitude of the modes. Crosses correspond to peaks not identified in the power spectrum.

small spacings $\overline{\delta v_{0,2}}$ and $\overline{\delta v_{1,3}}$ are $5.7 \pm 0.4 \mu \mathrm{Hz}$ and $7.5 \pm 0.4 \mu \mathrm{Hz}$ respectively.

The splitting of $l=2$ modes is displayed in Fig. 12 . Considering that such a splitting does not appear in modes $l=1$ and the fact that the visibilities of modes $m= \pm 2$ are higher than modes $m \pm 1$, we strongly suspect that the observed $l=2$ modes correspond to an azimuthal order $m= \pm 2$. The average splitting of $2.1 \pm 0.2 \mu \mathrm{Hz}$ leads to a rotational splitting $\Omega=0.53 \pm 0.05 \mu \mathrm{Hz}$ which corresponds to a rotational period of $22 \pm 2$ days in agreement with the very low magnetic activity found in Sect. 3. Modes $l=3$ do not appear to be split. This could indicate that we only observe modes $(l=3, m=0)$. 


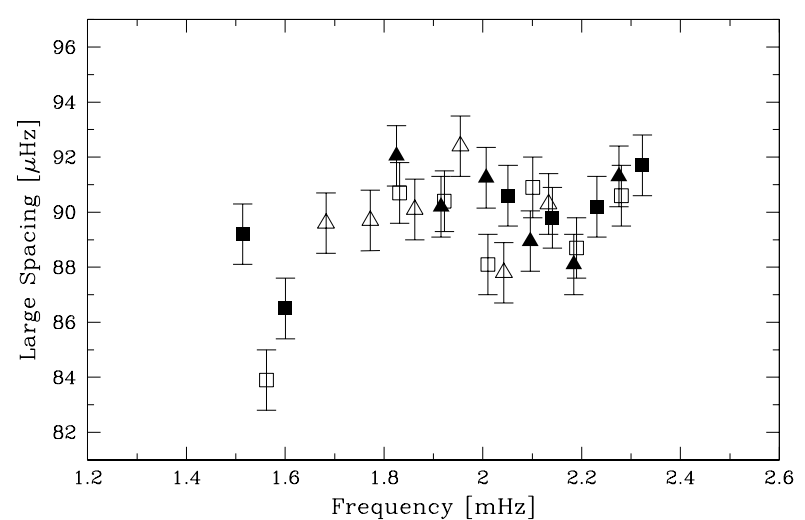

Fig. 11. Large spacing $\Delta v$ versus frequency for $p$-modes of degree $l=$ $0(\square), l=1(\mathbf{\square}), l=2(\mathbf{\Delta})$ and $l=3(\triangle)$.
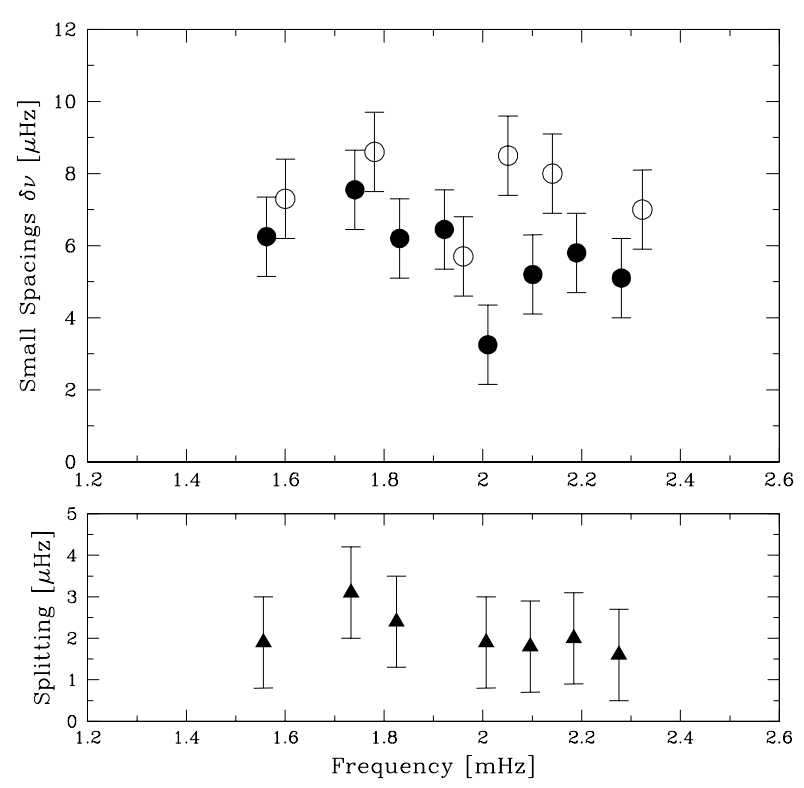

Fig. 12. (Top panel) Small spacings $\delta v_{0,2}(\bullet)$ and $\delta v_{1,3}(\circ)$ versus frequencies. (Bottom panel) Splitting of modes $l=2, m= \pm 2(\mathbf{\Lambda})$.

An alternative is that we observe modes $(l=3, m=-3)$. In this case modes $(l=3, m=+3)$ are expected to be at $\sim 11.1 \mu \mathrm{Hz}$ from the $l=1$ modes, hence completely merged with the $l=$ 1 side-lobes.

\section{Conclusions}

Our spectroscopic observations of HD 160691 first yield the discovery of a very light planetary companion $\left(14 M_{\oplus}\right)$ with a short period (9.55 days) (Santos et al. 2004b). Our analysis enforces the validity of this planetary companion excluding all sources of ambiguities. Our observations of HD 160691 also yield a clear detection of $p$-mode oscillations. Up to $43 p$-modes have been identified in the power spectrum between 1.3 and $2.5 \mathrm{mHz}$ with an average large spacing of $90 \mu \mathrm{Hz}$, an average small spacing of $5.7 \mu \mathrm{Hz}$, a rotational splitting of $0.53 \mu \mathrm{Hz}$ and an envelope amplitude of about $30 \mathrm{~cm} \mathrm{~s}^{-1}$. The identified $p$-mode frequencies are compared with stellar models in the companion paper (Bazot et al. 2005).

Acknowledgements. The authors wish to thank R. L. Gilliland for his helpful suggestions. We are grateful for the ESO staff support at the 3.6-m telescope. S.V. acknowledges a grant from Institut Universitaire de France. N.C.S. would like to thank the Swiss National Science Foundation and the Portuguese Fundação para a Ciência e Tecnologia for the scholarship.

\section{References}

Bazot, M., \& Vauclair, S. 2004, A\&A, 427, 965

Bazot, M., Vauclair, S., Bouchy, F., et al. 2005, A\&A, 440, 615

Bedding, T., \& Kjeldsen, H. 2003, PASA, 20, 203

Bouchy, F., Pepe, F., \& Queloz, D. 2001, A\&A, 374, 733

Bouchy, F., \& Carrier, F. 2002, A\&A, 390, 205

Bouchy, F., \& Carrier, F. 2003, Ap\&SS, 284, 21

Bouchy, F., Maeder, A., Mayor, M., et al. 2004, Nature, 432(7015), 2

Bouchy, F. 2005, in Dome C Astronomy/Astrophysics Meeting (EDP Sciences), EAS Publ. Ser., 14

Butler, R. P., Tinney, C. G., Marcy, G. W., et al. 2001, ApJ, 555, 410

Gonzalez, G. 1998, A\&A, 334, 221

Gozdziewski, K., Konacki, M., \& Maciejewski, A. J. 2003, ApJ, 594, 1019

Henry, T. J., Soderblom, D. R., Donahue, R. A., et al. 1996, AJ, 111, 439

Israelian, G., Santos, N. C., Mayor, M., et al. 2001, Nature, 411, 163

Jones, H. R. A., Butler, R. P., Marcy, G. W., et al. 2002, MNRAS, 337, 1170

Lomb, N. R. 1976, Ap\&SS, 39, 447

Mayor, M., Pepe, F., Queloz, D., et al. 2003, Messenger, 114, 20

Murray, N., \& Chaboyer, B. 2002, ApJ, 566, 442

Noyes, R. W., Hartmann, L. W., Baliunas, S. L., et al. 1984, ApJ, 279, 763

Pepe, F., Mayor, M., \& Rupprecht, G. 2002, Messenger, 110, 9

Queloz, D., Henry, G. W., Sivan, J. P., et al. 2001, A\&A, 379, 279

Santos, N. C., Israelian, G., \& Mayor, M. 2001, A\&A, 373, 1019

Santos, N. C., Mayor, M., Naef, D., et al. 2002, A\&A, 392, 215

Santos, N. C., Israelian, G., Mayor, M., et al. 2003, A\&A, 398, 363

Santos, N. C., Israelian, G., Mayor, M., et al. 2004a, A\&A, 415, 1153

Santos, N. C., Bouchy, F., Mayor, M., et al. 2004b, A\&A, 426, L19

Scargle, J. D. 1982, ApJ, 263, 835

Tassoul, M. 1980, ApJS, 43, 469

Vauclair, S. 2004, ApJ, 605, 874

Vaughan, A. H., Preston, G. W., \& Wilson, O. C. 1978, PASP, 90, 267 